\title{
Deriving Features of Reacting Hypersonic Flow from Gradients at a Curved Shock
}

\author{
H. G. Hornung* \\ California Institute of Technology, Pasadena, California 91125
}

DOI: $\underline{10.2514 / 1.39993}$

\begin{abstract}
The shock jump relations for gradients of flow variables are presented in the case of a uniform steady freestream with a curved shock that may trigger an endothermic or exothermic chemical reaction. The rich information that can be obtained from these is used in order to derive field information for flow variables as well as details about the sonic line. This presentation collects, reviews, and extends work that has been published previously in various places. Comparisons with experiment and with numerical computation are given in some cases. The gradient-jump relations are likely to be useful for higher-order numerical schemes that use shock fitting.
\end{abstract}

\section{Nomenclature}

$A=$ function of $M, \beta$, and $\gamma$

$a=$ dimensionless frozen speed of sound

$B=$ function of $M, \beta$, and $\gamma$

$C=$ function of $M, \beta$, and $\gamma$

$c=$ species mass fraction

$h=$ dimensionless specific enthalpy

$k=$ flow-plane shock curvature

$l=$ transverse shock curvature

$M=$ freestream Mach number

$n=$ number of species

$p=$ dimensionless pressure

$R=$ specific gas constant

$r=$ dimensionless reaction rate

$T=$ dimensionless absolute temperature

$u=$ dimensionless $x$ component of velocity

$V=$ magnitude of dimensionless velocity

$v=$ dimensionless $y$ component of velocity

$w=$ dimensionless $z$ component of velocity

$X=$ dimensionless distance along freestream direction

$x=$ dimensionless coordinate

$Y=$ dimensionless distance normal to freestream direction

$y=$ dimensionless coordinate

$z=$ dimensionless coordinate

$\alpha=$ angle between flow direction and feature direction

$\beta=$ shock angle

$\gamma=$ ratio of specific heats

$\delta=$ deflection angle

$\varepsilon=$ reaction rate coefficient

$\zeta=$ dimensionless $z$ component of vorticity

$\theta=$ dimensionless energy, also angle between feature and freestream direction

$\mu=$ Mach angle

$\rho=$ dimensionless density

$\phi=$ angle between shock and feature direction

Subscripts

$i \quad=\quad$ species number

$s=$ switch angle

Presented as Paper 4340 at the Theoretical Fluid Mechanics Conference, Seattle, WA, 23-26 June 2008; received 23 July 2008; revision received 25 July 2008; accepted for publication 7 November 2008. Copyright @ 2008 by the American Institute of Aeronautics and Astronautics, Inc. All rights reserved. Copies of this paper may be made for personal or internal use, on condition that the copier pay the $\$ 10.00$ per-copy fee to the Copyright Clearance Center, Inc., 222 Rosewood Drive, Danvers, MA 01923; include the code 0001-1452/10 \$10.00 in correspondence with the CCC.

*Kelly Johnson Professor of Aeronautics, Emeritus, Graduate Aeronautical Laboratories. Lifetime Fellow AIAA.

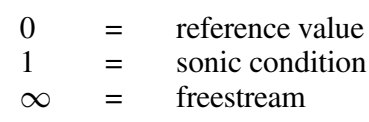

Superscript

, = dimensional value

\section{Introduction}

$\mathbf{I}^{\mathrm{N}}$ $\mathrm{N}$ A broad class of situations shock waves may be modeled as surfaces across which flow variables change discontinuously. The jumps of the values of flow variables across the shock are obtained by applying the integrated conservation equations to a control volume that straddles the shock. If the shock wave under consideration is curved, or if it accelerates, or if a chemical reaction is triggered by it, or if the upstream medium is nonuniform, the flow variables downstream of the shock will be spatially and/or temporally nonuniform. It is possible to determine the gradients or rates of change of flow variables at the downstream side of the shock by solving the differential form of the conservation equations. In other words, it is possible to derive jump conditions for the gradients, or rates of change of the flow variables in terms of the curvature or acceleration of the shock, the nonuniformity of the freestream conditions, and the reaction rate.

A number of textbooks have treated parts of this topic (e.g., Hayes and Probstein [1] and Oswatitsch [2]) and several publications treat different aspects (e.g., Lighthill [3], Munk and Prim [4], Gerber and Bartos [5], Chen and Gurtin [6] ], Gilinskii and Chernyi [7], Clarke []ㅡ, and Mölder $[\underline{9}, 10]$. The present author has worked on this problem over a number of years, concentrating on the case of a curved shock in a steady, uniform freestream including the following: Hornung [11] to treat a thin reacting layer after a curved shock with matched asymptotic expansions, Hornung [12] (with corrections by Kaneshige and Hornung [13]) to obtain streamline curvature and sonic line slope for curved shocks with reactions, and Hornung [14] to discuss the density field downstream of a curved shock with chemical reaction.

In this paper some of the previous work is put into a more complete and self-consistent form, and some new features are added. To do the former in a way that gathers all the derivations into one place, it has been necessary to go over previously covered ground again at the beginning of the argument. The presentation is restricted to the case of a steady, uniform freestream.

A particular goal of this work is to proceed in the manner of Hornung [14], that is, to use the behavior of the values and gradients of a flow variable $a t$ the shock in order to speculate about the field of this variable in the region downstream of the shock. At least in this sense, namely the boldly speculative approach (but in others too) this work attempts to recall the spirit of Norm Malmuth. 


\section{Shock Jump Conditions for Gradients}

\section{A. Coordinates, Variables, and Gas Properties}

Consider a continuously curved shock wave in a uniform, steady freestream characterized by velocity $V_{\infty}^{\prime}$ and density $\rho_{\infty}^{\prime}$. Our interest is to determine the gradients of flow quantities at the shock in terms of the shock angle and curvature and the freestream and gas properties, and we therefore focus on a particular point on the shock. Accordingly, the origin of the shock-aligned, orthogonal, curvilinear coordinates $x^{\prime}, y^{\prime}$, and $z^{\prime}$ is chosen to lie at this point of interest. The $y^{\prime}$ coordinate is chosen to be normal to the shock.

Choose the $x^{\prime} y^{\prime}$ plane to be the plane defined by the freestream direction and the $y^{\prime}$ coordinate. With this choice, the velocity component in the $z^{\prime}$ direction and its gradients in the $x^{\prime} y^{\prime}$ plane are zero both on the upstream and immediate downstream point of the shock. Thus, a suitable name for this plane is the "flow plane." Let the shock curvatures at this point be $k^{\prime}$ in the flow plane and $l^{\prime}$ in the $y^{\prime} z^{\prime}$ plane. The velocity components are $u^{\prime}, v^{\prime}, w^{\prime}$ in the $x^{\prime}, y^{\prime}$, and $z^{\prime}$ directions, respectively. Let the shock and deflection angles in the flow plane be $\beta$ and $\delta$ as shown in Fig. 1 .

Introduce dimensionless variables defined by

$$
\begin{gathered}
h=h^{\prime} / V_{\infty}^{\prime 2}, \quad p=p^{\prime} / \rho_{\infty}^{\prime} V_{\infty}^{\prime 2}, \quad v=v^{\prime} / V_{\infty}^{\prime} \\
\rho=\rho^{\prime} / \rho_{\infty}^{\prime}, \quad y=y^{\prime} k_{0}^{\prime}, \quad k=k^{\prime} / k_{0}^{\prime}
\end{gathered}
$$

where $h, p, \rho$, and $v$ are dimensionless specific enthalpy, pressure, density, and $y$ velocity, and $k_{0}^{\prime}$ is a convenient reference value of the shock curvature. The other components of vectors are made dimensionless in the same way as the components shown.

The gas is supposed to obey caloric and thermal equations of state of the forms

$$
\begin{aligned}
& h=h\left(p, \rho, c_{i}\right) \\
& T=T\left(p, \rho, c_{i}\right)
\end{aligned}
$$

in which $T$ is the dimensionless absolute temperature $R T^{\prime} / V_{\infty}^{\prime 2}$, with the specific gas constant $R$, and the $c_{i} \mathrm{~s}$ are the mass fractions of the $n$ constituent species of the gas, with $i$ taking values 1 through $n$.

Because the mass fractions must satisfy the identity

$$
\sum_{i=1}^{n} c_{i}=1
$$

the number of mass fractions that are independent is one less than the total number $n$ of components present. It is usually convenient to choose $c_{1}$ as a dependent variable and the other $c_{i}$ s as independent variables. Thus,

$$
\mathrm{d} h=h_{\rho} \mathrm{d} \rho+h_{p} \mathrm{~d} p+\sum_{i=2}^{n} h_{c_{i}} \mathrm{~d} c_{i}
$$

where the subscripts (other than $i$ ) denote partial differentiation.

\section{B. Equations and Jump Conditions}

To determine the gradients of physical properties of the flow at the shock wave, it is necessary to solve the differential equations of motion for the components of the gradients. With our flow-planealigned coordinate system $w$ and all $z$ derivatives except $w_{z}$ are zero. So, also, are the $x$ and $y$ derivatives of $w$. This means that all terms in the $z$ component of the momentum equation are zero. Furthermore, the terms involving $w$ and its derivatives disappear in the other two components of the momentum equation. Recall that we are only interested in the gradients at the shock, that is, at $y=0$. This allows us to put terms like $1-k y$ and $1-l y$ that arise in our curvilinear coordinates equal to one. The resulting equations are

$$
\begin{gathered}
u u_{x}+v u_{y}-k u v+p_{x} / \rho=0 \\
u v_{x}+v v_{y}+k u^{2}+p_{y} / \rho=0 \\
h_{p} p_{y}+h_{\rho} \rho_{y}+\sum_{i=2}^{n} h_{c_{i}} c_{i y}+v v_{y}+u u_{y}=0 \\
h_{p} p_{x}+h_{\rho} \rho_{x}+u u_{x}+v v_{x}=0 \\
(\rho u)_{x}-(k+l) \rho v+\rho l \sin \beta+(\rho v)_{y}=0
\end{gathered}
$$

The energy equation has been written in $x$ and $y$ differentiated form for convenience in solving the equations for the $y$ derivatives of the flow variables. Note that the only place where $l$ appears is in two terms in the continuity equation. The second of these arises because $w_{z}=l \sin \beta$.

The shock jump relations

$$
\begin{gathered}
p-p_{\infty}=\sin ^{2} \beta(1-1 / \rho) \\
c_{i}=c_{i \infty}
\end{gathered}
$$

$$
v=\sin \beta / \rho
$$

$$
2\left(h-h_{\infty}\right)=\sin ^{2} \beta\left(1-1 / \rho^{2}\right)
$$

$$
u=\cos \beta
$$

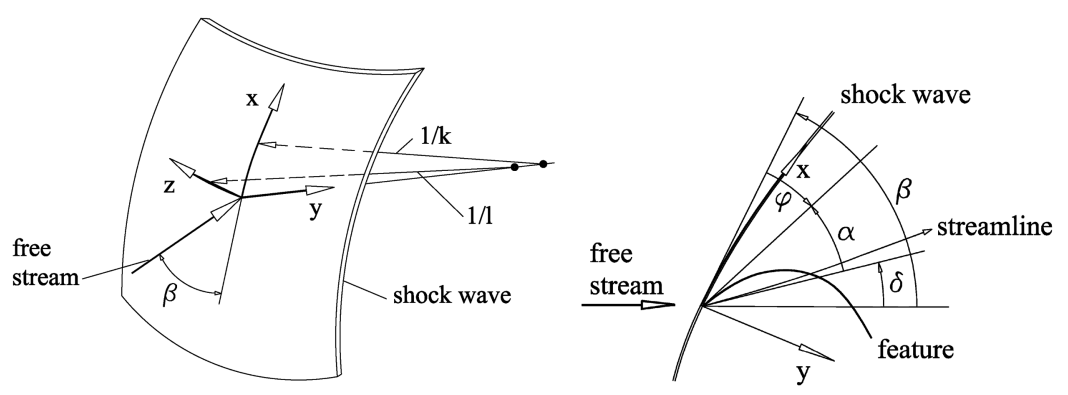

Fig. 1 The left shows a sketch of a three-dimensional curved shock wave and orientation of coordinate system. $k$ and $l$ are the flow-plane and transverse dimensionless shock curvatures. The right shows a sketch of the flow plane, also defining angles that will be used in later sections. The heavy line is some feature, such as the sonic line or a contour of some variable like density, entropy, or pressure. The angles $\phi, \alpha$, and $\theta=\alpha+\delta$ measure the direction of the feature at the shock wave relative to the shock, relative to the streamline, and relative to the freestream direction, respectively. $\delta$ is the deflection angle of the streamline at the shock. 


$$
\rho=\frac{\gamma+1}{\gamma-1+2 /\left(M^{2} \sin ^{2} \beta\right)}
$$

are also needed. Here $\gamma$ is the ratio of specific heats, and $M$ is the freestream Mach number. The expression for the density ratio across the shock, as shown in Eq. (13), is written for a constant- $\gamma$ gas. This is permissible in a reacting flow situation if the shock jump relations are taken to apply to the jump from the freestream conditions to the conditions downstream of the shock before any reactions take place, that is, to a jump that does not involve a change of composition, as is made clear by Eq. (9).

\section{Partial Derivatives at the Shock}

The problem of determining the gradients of the flow variables at the shock consists of solving Eqs. (3-5) and (7) for the $y$ derivatives and determining the $x$ and $z$ derivatives (along the shock) by differentiating the shock jump relations with respect to $x$ and $z$.

Differentiation of the shock jump conditions with respect to $x$ introduces the shock curvature $\beta_{x}=-k$ :

$$
\begin{gathered}
\frac{u_{x}}{k}=\sin \beta \\
\frac{\rho_{x}}{k}=-\frac{4 \rho^{2} \cos \beta}{(\gamma+1) M^{2} \sin ^{3} \beta} \\
\frac{p_{x}}{k}=-2 \sin \beta \cos \beta\left(1-\frac{1}{\rho}\right)+\frac{\sin ^{2} \beta}{\rho^{2}} \frac{\rho_{x}}{k} \\
\frac{v_{x}}{k}=-\frac{\cos \beta}{\rho}-\frac{\sin \beta}{\rho^{2}} \frac{\rho_{x}}{k}
\end{gathered}
$$

By choosing the $x y$ plane to be the flow plane the only nonzero $z$ derivative is $w_{z}=l \sin \beta$ (already used in the continuity equation).

To simplify the notation, introduce

$$
r=v \sum_{i=2}^{n} h_{c_{i}} c_{i y}=\sum_{i=2}^{n} h_{c_{i}} \frac{\mathrm{d} c_{i}}{\mathrm{~d} t}
$$

where $\mathrm{d} / \mathrm{d} t$ constitutes the substantial derivative with respect to time. Solving Eqs. (3-ㄱ) for the $y$ derivatives yields

$$
\begin{gathered}
p_{y} F=\frac{\rho r}{v}+l \rho^{2} h_{\rho}\left(1-\frac{\sin \beta}{v}\right) \\
-k \rho\left[u\left(1-\frac{\rho h_{\rho}}{v^{2}}\right) K+\frac{u}{v} E+\rho \frac{h_{\rho}}{v} L\right] \\
u_{y}=-\frac{k E}{v} \\
v v_{y} F=-\frac{r}{v}-l \rho h_{\rho}\left(1-\frac{\sin \beta}{v}\right)+k\left[\rho u h_{p} K+\frac{u}{v} E+\rho \frac{h_{\rho}}{v} L\right]
\end{gathered}
$$

$$
\begin{aligned}
\rho_{y} F & =\frac{\rho r}{v^{3}}+l \rho\left(1-\rho h_{p}\right)\left(1-\frac{\sin \beta}{v}\right) \\
- & \frac{k \rho}{v^{2}}\left[\rho u h_{p} K+\frac{u}{v} E+\left(1-\rho h_{p}\right) L v\right]
\end{aligned}
$$

where

$$
\begin{gathered}
K=\frac{v_{x}}{k}+u \\
L=\frac{(\rho u)_{x}}{\rho k}-v \\
E=\frac{p_{x}}{\rho k}+u \frac{u_{x}}{k}-u v
\end{gathered}
$$

$$
F=1-\rho\left(\frac{h_{\rho}}{v^{2}}+h_{p}\right)
$$

\section{Discussion of Results so Far}

At this point it is worth taking a closer look at these results. Because we are interested in the point at the shock before chemical reactions occur, we can write

$$
h_{p}=\gamma /[\rho(\gamma-1)], \quad h_{\rho}=-\gamma p /\left[\rho^{2}(\gamma-1)\right]
$$

Also, the dimensionless freestream pressure and enthalpy may be written as

$$
p_{\infty}=1 /\left(\gamma M^{2}\right), \quad h_{\infty}=1 /\left[(\gamma-1) M^{2}\right]
$$

Thus, $K, L, E$, and $F$ as well as all the coefficients of $r, l$, and $k$ in Eqs. (14-21) are explicitly known functions of $M, \gamma$, and $\beta$, so that Eqs. $(\overline{18}-\overline{21})$ may be written formally as

$$
\begin{gathered}
p_{y}=A_{(p)}(M, \gamma, \beta) r+B_{(p)}(M, \gamma, \beta) l+C_{(p)}(M, \gamma, \beta) k \\
u_{y}=C_{(u)}(M, \gamma, \beta) k
\end{gathered}
$$

$$
v_{y}=A_{(v)}(M, \gamma, \beta) r+B_{(v)}(M, \gamma, \beta) l+C_{(v)}(M, \gamma, \beta) k
$$

$$
\rho_{y}=A_{(\rho)}(M, \gamma, \beta) r+B_{(\rho)}(M, \gamma, \beta) l+C_{(\rho)}(M, \gamma, \beta) k,
$$

where the $A \mathrm{~s}, B \mathrm{~s}$, and $C \mathrm{~s}$ are explicitly known functions. As expected, each $y$ derivative has contributions arising from the chemical reaction rate $r$, the flow-plane shock curvature $k$, and the transverse shock curvature $l$. The same applies to the $x$ derivatives in Eqs. (14-17) except that they have only a $k$ contribution. Because all the $z$ derivatives except $w_{z}$ are zero, the gradient vectors of all flow quantities except those of $w$ lie in the flow plane. The gradient vector of $w$ is perpendicular to the flow plane.

It is worth noting here that $r$ is the rate at which chemical reactions change the specific enthalpy $h$ of an element of fluid. Thus, the flow variables are not explicitly dependent on the details of the possibly complicated chemistry among possibly many different species, but rather they respond to chemistry only because of its effect on the enthalpy.

Effectively our results constitute shock jump conditions for gradients of the flow quantities in terms of (in addition to $M, \gamma$, and $\beta$ ) the reaction rate at the shock $r$ and the shock curvatures $k$ and $l$. They are valid for a general shock point in three-dimensional, reacting, steady flow with a uniform freestream.

\section{Example Shock-Shape and Reaction Model}

\section{A. Shock Shape}

The results for the three-dimensional case may be useful in numerical investigations. However, for determining features of 


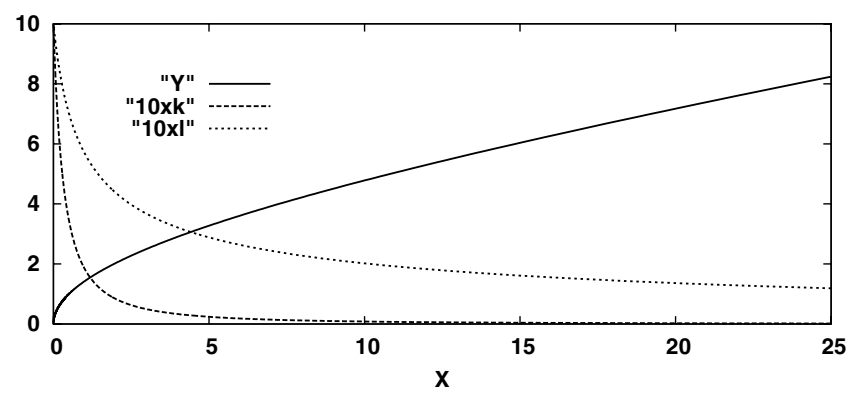

Fig. 2 Shape of hyperbolic shock for $M=6, Y(X)$, and corresponding curvature $10 \times k$. For the axisymmetric case the transverse curvature is shown as $10 \times l$.

flowfields downstream of a shock, it is more useful to consider twodimensional (plane or axisymmetric) flows. In steady flow, curved shock waves often occur as detached bow waves in flow over bodies. For a flow with a plane or an axis of symmetry, a very good approximation to the bow shock shape is the hyperbola

$$
Y=\tan \mu \sqrt{X\left(X+\frac{2}{\tan ^{2} \mu}\right)}
$$

in which $X$ is the distance along the freestream direction measured from the normal-shock point made dimensionless with the radius of curvature at the normal-shock point, $Y$ is the distance normal to $X$ similarly normalized, and $\mu=\arcsin (1 / M)$ is the Mach angle. This shock shape makes a smooth transition from a finite flow-plane curvature at $X=0$ to a straight shock at the Mach angle at $X=\infty$. For such a hyperbolic shock, the shock angle $\beta$ may be obtained from

$$
\tan \beta=\tan \mu \frac{1+1 /\left(X \tan ^{2} \mu\right)}{\sqrt{1+2 /\left(X \tan ^{2} \mu\right)}}
$$

The flow-plane curvature (normalized by the curvature at $X=0$ ) is

$$
k=\left(1+2 X / \cos ^{2} \mu+X^{2} \tan ^{2} \mu / \cos ^{2} \mu\right)^{-3 / 2}
$$

(See Fig. 2.) In axisymmetric flow, the flow plane is the meridional plane, and the dimensionless transverse curvature is $l=\cos \beta / Y$. At $X=0, k=l=1$. Thus, for a given $M$, the shock-shape parameters required to determine the gradients at a point on the $\operatorname{shock}(\beta, k$, and $l)$ are known. All the examples treated in the following are calculated for the case $M=6$. Also, $\gamma=1.4$ is assumed in all examples.

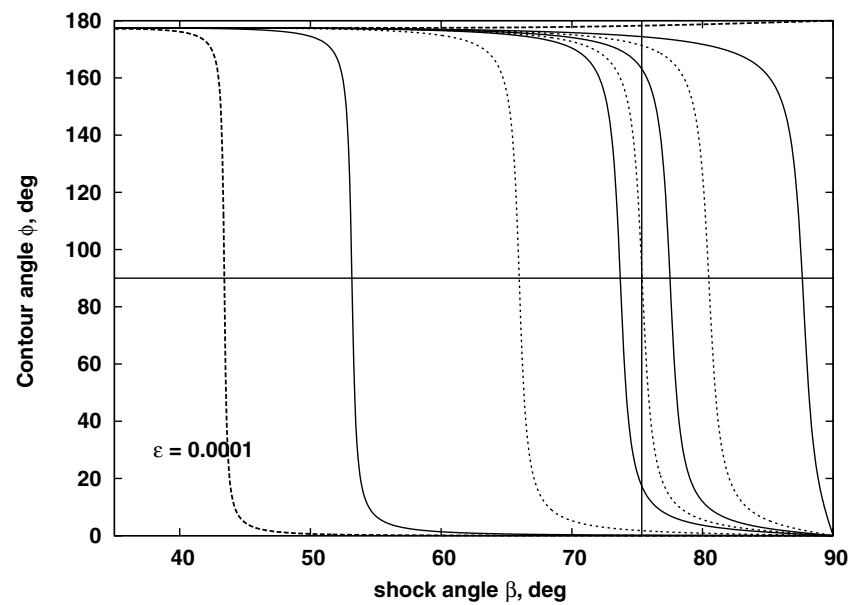

\section{B. Reaction Model}

To compute numerical values it is also necessary to introduce a model for the reaction rate. Although the gradient jump conditions are valid for both endothermic and exothermic reactions, the chemistry model chosen is very simple, tailored to the endothermic reaction situation encountered in dissociating hypersonic flow. In the following sections the same model is assumed to apply also for exothermic flow, though we deal mainly with the endothermic case. The model is assumed to be of the Arrhenius form (without the recombination term, because only dissociation occurs at the shock).

$$
r=\frac{\theta}{\varepsilon} \exp (-\theta \rho / p)
$$

where $\theta$ is the dissociation energy and $1 / \varepsilon$ is a measure of the rate constant. This ensures that $r$ has the right dependence on temperature and therefore on $\beta$. All the examples that are treated in the following are calculated for the case $\theta=0.95$.

\section{Density Field}

\section{A. Angle of Density Contour}

The ratio $\rho_{y} / \rho_{x}$ (from Eqs. (15) and (21) gives the tangent of the direction of the density gradient at the shock relative to the shock. The direction of the line of constant density (or density contour) is normal to this, thus giving the angle $\phi$ between the density contour and the shock (see Fig. 1). The result is shown in Fig. 3 in the form of plots of $\phi$ vs. $\beta$, with $\varepsilon$ as a parameter, for both plane and axisymmetric flow. These curves exhibit some interesting features. Consider first the frozen-flow light broken curve for the plane case (Fig. 3 left). At small shock angles $\beta, \phi$ is quite close to $180 \mathrm{deg}$, so that the density contour leaves in a direction pointing roughly toward the stagnation point. Then, in a small range of shock angles around $75 \mathrm{deg}, \phi$ changes to a value close to zero, indicating that the density contour leaves the shock in a direction pointing roughly away from the stagnation point. Thus, in the small range around $75 \mathrm{deg}$, the density contour direction switches through $90 \mathrm{deg}$, which means that there must be a saddle point in the density field at that point. With the parameters of Fig. 3 the location of this saddle point is at $\beta=$ $75.35 \mathrm{deg}$ for plane flow. Figure $\underline{3}$ shows a vertical line at this point. In axisymmetric flow the angle is $68.84 \mathrm{deg}$.

To illustrate this feature, a computation of the inviscid flow over a circular cylinder was made using the Euler equations in the Amrita environment of Quirk [15]. The computation used a kappa-muscl HLLE scheme with adaptive mesh refinement. The coarse grid of $300 \times 60$ is refined in two levels by a factor of three each in places where the density gradient exceeds a chosen threshold. Thus, the effective resolution is equivalent to using a $2700 \times 540$ grid. The grid

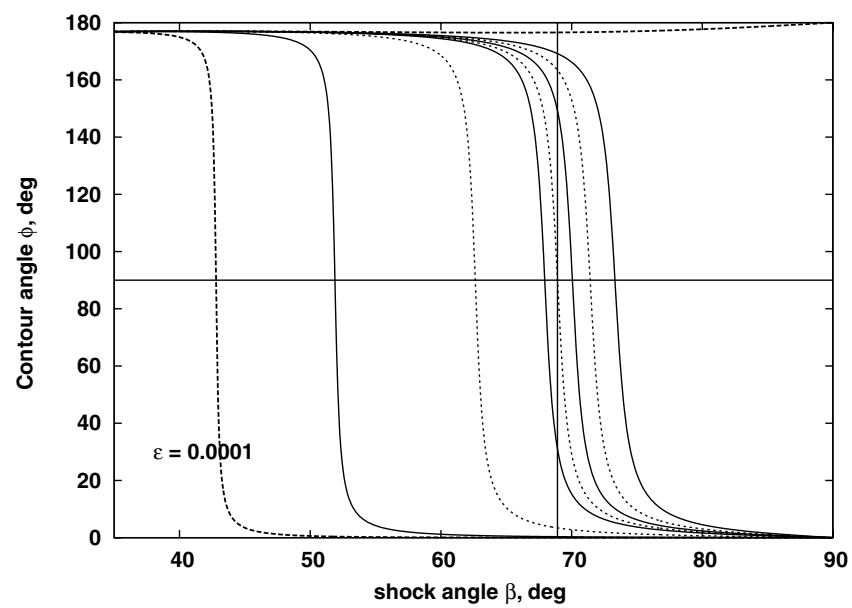

Fig. 3 Angle between the shock and density contour. The left shows plane flow. The right shows axisymmetric flow. The curves show the behavior of $\phi(\beta)$ for different values of $\varepsilon=10^{-4}, 10^{-3}, 10^{-2}, 10^{-1}, 10,-10^{-1},-10^{-2},-10^{-3}$, and $-10^{-4}$, in heavy-broken, full, broken, light-full, light-broken, lightfull, broken, full, and heavy-broken lines, respectively, so that the leftmost heavy-broken curve corresponds to a high dissociation rate, the light-broken curve corresponds to frozen flow $(r=0)$, and the curves to the right of the light-broken curve correspond to exothermic reaction $(r<0)$. Hyperbolic shock, $M=6, \gamma=1.4, \theta=0.95$. 


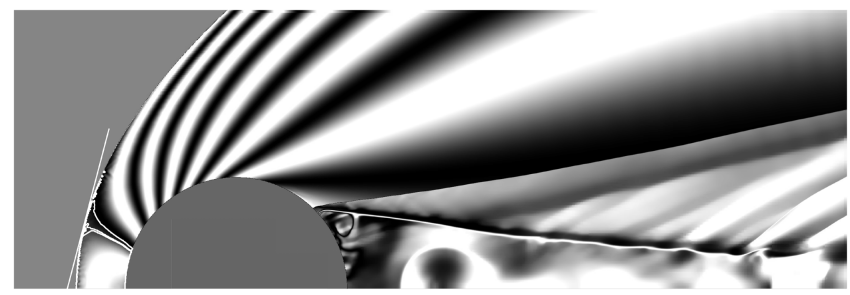

Fig. 4 Computational interferogram, in which the fringe shift is proportional to density change, of frozen flow over a circular cylinder. $M=6, \gamma=1.4$. Note the saddle point in the fringes. To check the location of this saddle point, three lines of constant density are shown in white at values near those in the vicinity of the saddle point. Also a straight line tangent to the shock is drawn at an angle of $75.35 \mathrm{deg}$ to show how closely the theoretical value agrees with the computation.

is Cartesian, and the body boundary conditions are inserted by means of a level set. The result of this computation is shown in Fig. 4.

The hyperbolic shock is a very good approximation to the shock shape that occurs in the flow over a circular cylinder or over a sphere, for a wide range of freestream Mach numbers. An approximation for the relative location of the normal-shock point and the stagnation point in such flows has been given for the hyperbolic shock by Billig [16]. The hyperbolic shock shape is also a good approximation for flow over other nonlifting bodies with detached bow shocks.

\section{B. Saddle Point Location and Dissociation Rate}

A second interesting feature of Fig. 3 is that the location of the point at which the switch of $\phi$ from near $180 \mathrm{deg}$ to near 0 deg occurs moves to smaller shock angles $\beta$ as the dissociation rate is increased, and this switch angle $\beta_{s}$ is quite sensitive to the dissociation rate, slightly more so in plane than in axisymmetric flow. This behavior can be observed very nicely in experimental interferograms of flow over a circular cylinder, shown in Fig. 5. The fringes in these interferograms are almost exactly lines of constant density. The location of the saddle points in these interferograms show very dramatically that these are flows with fast dissociation rates, very distinct from the frozen flow of Fig. 4 with $\beta_{s}=75.35 \mathrm{deg}$. A number of authors have computed the flows shown in Fig. 5 with nonequilibrium chemistry, see, for example, Candler [17] and Klomfass [18].

\section{Sonic Line Behavior}

An excellent discussion of the behavior of the sonic line in nonreacting blunt-body flows is given by Hayes and Probstein [1]. What is new relative to [1] in the following is the inclusion of the effects of finite $r$. Hayes and Probstein [1] point out that, for frozen plane flow, a closed-form solution has been found for the sonic line direction at the shock that is independent of shock curvature. For finite $r$, the shock curvature does have an effect.

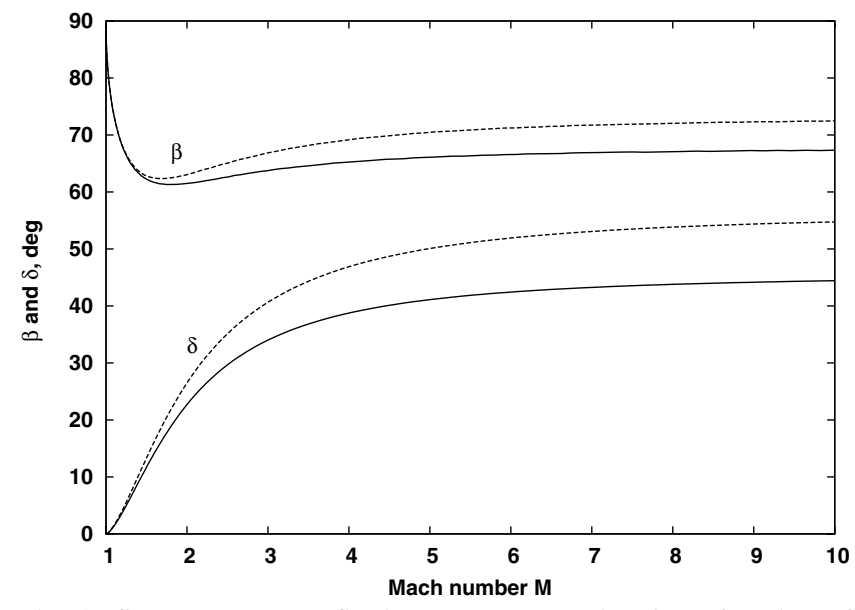

Fig. 6 Shock angle and deflection angle at the sonic point as functions of the freestream Mach number for $\gamma=1.4$ (full) and 1.2 (broken).

\section{A. Sonic Point on the Shock}

First, it is necessary to determine the location of the sonic point on the shock. This is nothing new, but we need it for the next step. Figure 6 shows the shock angle $\beta_{1}$ at which the flow downstream of the shock is sonic as a function of freestream Mach number for two values of $\gamma=1.4$ and 1.2.

\section{B. Direction of the Sonic Line at the Shock}

To determine the sonic line slope, consider the energy equation, for our isoenergetic flow and the model gas, in the form

$$
\frac{V^{2}}{2}+\frac{a^{2}}{\gamma-1}+h_{\text {chem }}=h_{0}
$$

where $h_{\text {chem }}$ is chemically stored specific energy, $V^{2}=u^{2}+v^{2}$ and $a$ is the frozen speed of sound. $V=a$ along the sonic line, for which we can therefore write

$$
\frac{\gamma+1}{2(\gamma-1)} V^{2}+h_{\text {chem }}=h_{0}
$$

Differentiating this along the sonic line, and recalling that $c_{i x}=0$

$$
V_{x} \cos \phi+V_{y} \sin \phi+\sin \phi \frac{\gamma-1}{\gamma+1} \frac{r}{V v}=0
$$

Here, $\phi$ is the angle between the sonic line and the shock, see Fig. 2 for notation. Thus,

$$
\tan \phi=\frac{-V_{x}}{V_{y}+\frac{\gamma-1}{\gamma+1} \frac{r}{V v}}
$$
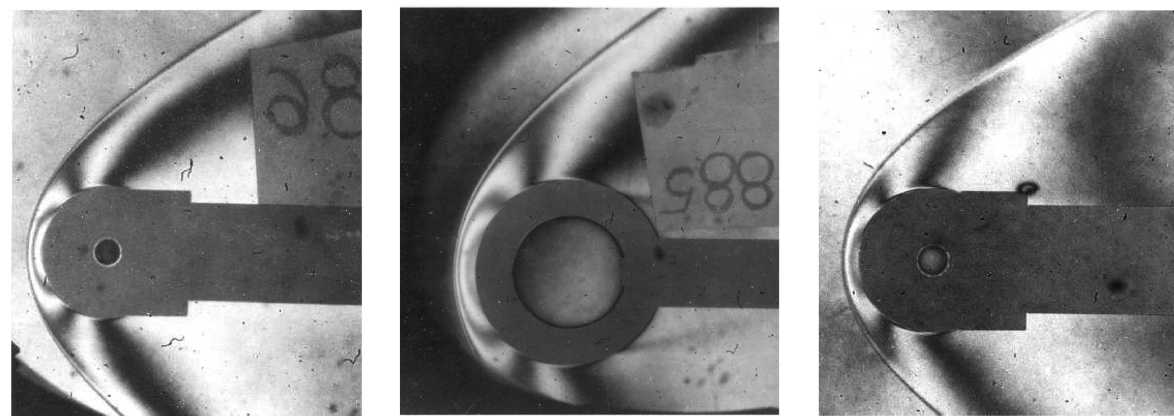

Fig. 5 Infinite-fringe interferograms of flows over a circular cylinder in the presence of dissociation from experiments performed in the T3 shock tunnel by Hornung [13]. The left shows nitrogen flow over a $25.4 \mathrm{~mm}$ diameter cylinder at a speed of $5.59 \mathrm{~km} / \mathrm{s}$, density $0.005 \mathrm{~kg} / \mathrm{m}^{3}$. The value of $\beta_{\mathrm{s}}$ is approximately $49 \mathrm{deg}$, corresponding to $\varepsilon \simeq 1.5 \times 10^{-4}$ The middle shows the same freestream conditions, but with a cylinder diameter of $50 \mathrm{~mm}$, corresponding to a doubling of $r$. This is nicely reflected in the change of the value of $\beta_{s}$ from 49 to $45 \mathrm{deg}$. The right shows carbon dioxide flow over the same cylinder as in the left picture. $V_{\infty}^{\prime}=3.6 \mathrm{~km} / \mathrm{s}, \rho_{\infty}^{\prime}=0.0016 \mathrm{~kg} / \mathrm{m}^{3}$. In this case $r$ is so large that the saddle is moved to such small shock angles that it does not appear within the field of view. 

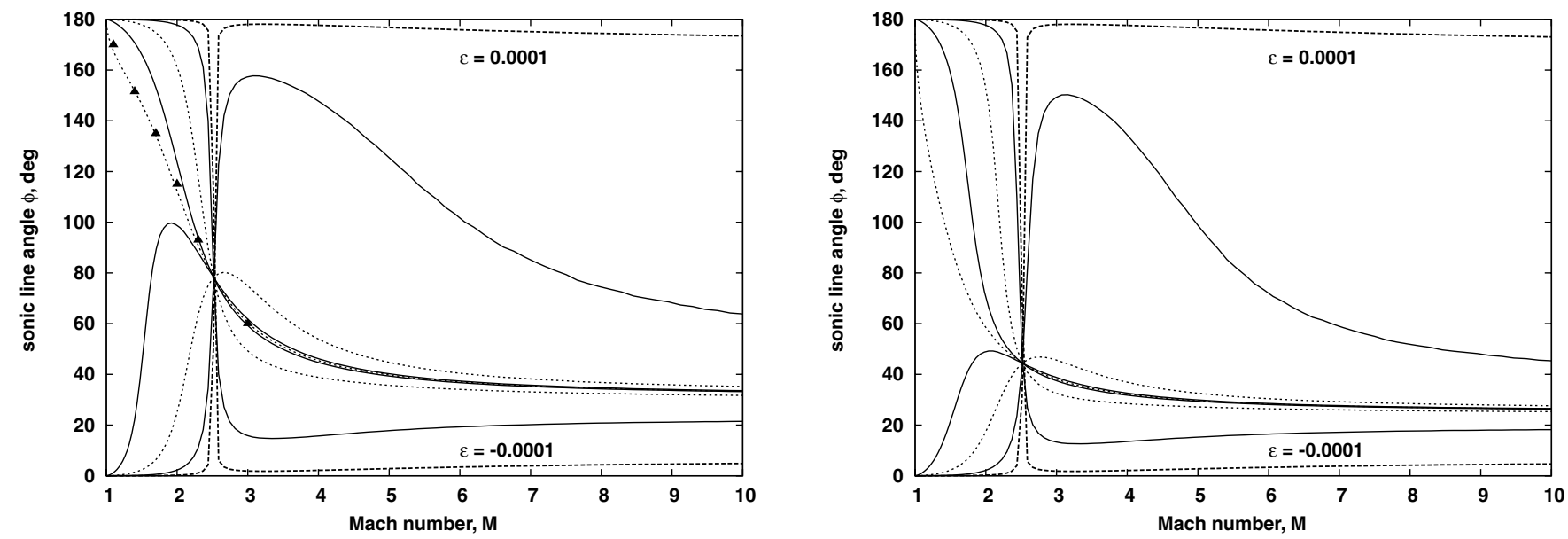

Fig. 7 The left shows the sonic line slope at the shock for plane, and the right shows the axisymmetric flow. $\gamma=1.4$ and $\theta=0.95$. The different line styles are for different values of $\varepsilon$, see Fig. 3 for key. The triangular symbols indicate values of $\phi$ obtained by numerical computation of frozen-flow over wedges.

Figure $\underline{7}$ shows $\phi$ as a function of $M$ for $\gamma=1.4$ for both plane and axisymmetric flow. It shows that, at high Mach number, endothermic reactions cause $\phi$ to increase, and vice versa, but these trends are reversed at low Mach number. The reversal occurs at a particular Mach number, which, for the value of $\gamma$ chosen, is approximately 2.5. This is the point where the reaction rate term in $V_{y}$ just cancels the one that occurs explicitly in the denominator of Eq. (37). This condition occurs at

$$
\frac{p}{\rho v^{2}}=\frac{\gamma^{2}-\gamma+2}{\gamma^{2}-1}
$$

Using Eqs. (8), (10), and (13) to express $p, v$, and $\rho$ in terms of $M, \gamma$, and $\beta$ then leads [for the particular value of $\beta=\beta_{1}(\gamma, M)$ at the sonic point] to a particular value of $M(\gamma)$ at which the reaction rate does not influence the sonic line slope.

\section{Discussion}

Because the qualitative behavior of the sonic line slope near the shock is quite similar in plane and axisymmetric flow, we focus on the left part of Fig. 7 for plane flow. (It should be pointed out here that the sonic line behavior near the body is quite different in the two geometries, see Hayes and Probstein [1]). First consider the low supersonic range. Here $\phi$ decreases from $180 \mathrm{deg}$ at $M=1$ to about $146 \mathrm{deg}$ at $M=1.5$ in the case of nonreacting or frozen flow (light broken curve). In this range it takes only a very slight rate of heat removal by an endothermic reaction to cause a dramatic reduction of $\phi$ to values close to 0 (see lower light full line for $\varepsilon=0.1$ ). Small exothermic reaction rates do not cause such dramatic changes (see

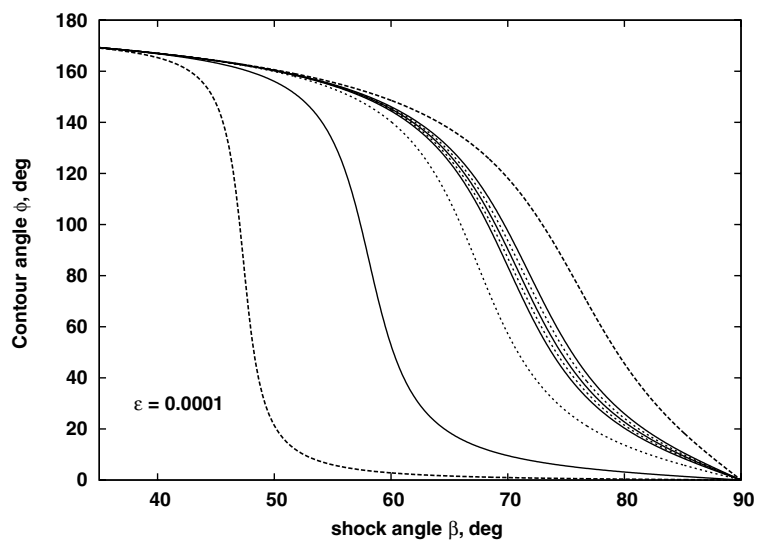

upper light full curve for $\varepsilon=-0.1$ ). Because the behavior of the sonic line is intimately related to many features of external and internal flows, this dramatic effect of heat addition or removal by chemical or other rate processes on the sonic line behavior seems to be a promising topic.

Another important effect occurs as a consequence of endothermic reaction at high Mach number. For example, at $M=6$, a change of $\varepsilon$ from 0.1 to 0.0001 causes $\phi$ to change from 37 to $176 \mathrm{deg}$. This change has been shown to have a very large effect on the bow-shock detachment process in flows over wedges (see Hornung and Smith [19]) and cones (see Hornung and Leyva [20]). This is one of the largest effects of dissociative nonequilibrium processes in hypersonic flow.

\section{Check by Computation of Frozen Flow}

For the purpose of checking some of these results for consistency, numerical computations were made of frozen flows over wedges at various Mach numbers. The wedge angles were chosen to be large enough to cause the shock to be detached. The angle $\phi$ was measured from the computed results, and these values are plotted as triangular symbols in Fig. 7.

\section{Pressure Field}

In a similar way as in the density field, the pressure contours downstream of a hyperbolic shock also exhibit a saddle point at the shock. However, the swing of $\phi$ from near $180 \mathrm{deg}$ to near 0 deg is less abrupt and much less sensitive to the reaction rate, see Fig. 8. This lack of sensitivity to $r$ is particularly pronounced in the case of

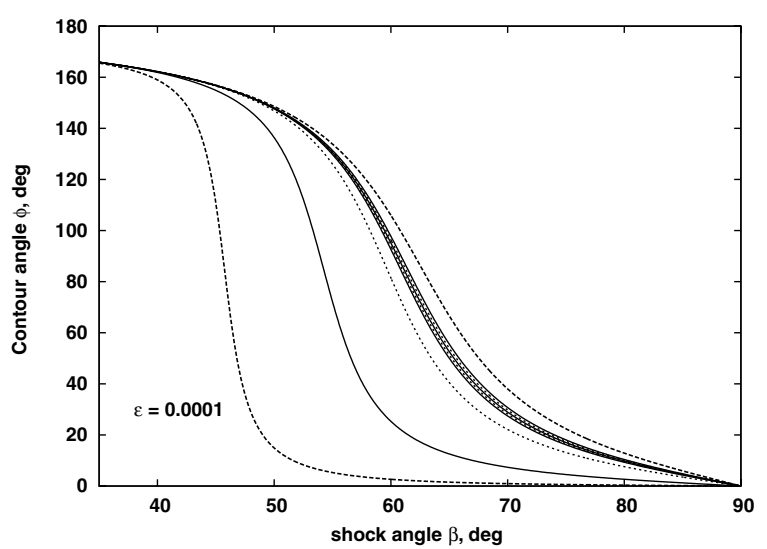

Fig. 8 Angle between shock and pressure contour. The left shows the plane, and the right shows the axisymmetric flow. As before, the curves show the behavior of $\phi(\beta)$ for different values of $\varepsilon=10^{-4}, 10^{-3}, 10^{-2}, 10^{-1}, 10,-10^{-1},-10^{-2},-10^{-3}$, and $-10^{-4}$, in the heavy-broken, full, broken, light-full, lightbroken, light-full, broken, full, and heavy-broken lines, respectively, so that the leftmost heavy-broken curve corresponds to a high dissociation rate, the light-broken curve corresponds to frozen flow $(r=0)$, and the curves to the right of the light broken curve correspond to exothermic reaction $(r<0)$. Hyperbolic shock, $M=6, \gamma=1.4, \theta=0.95$. 


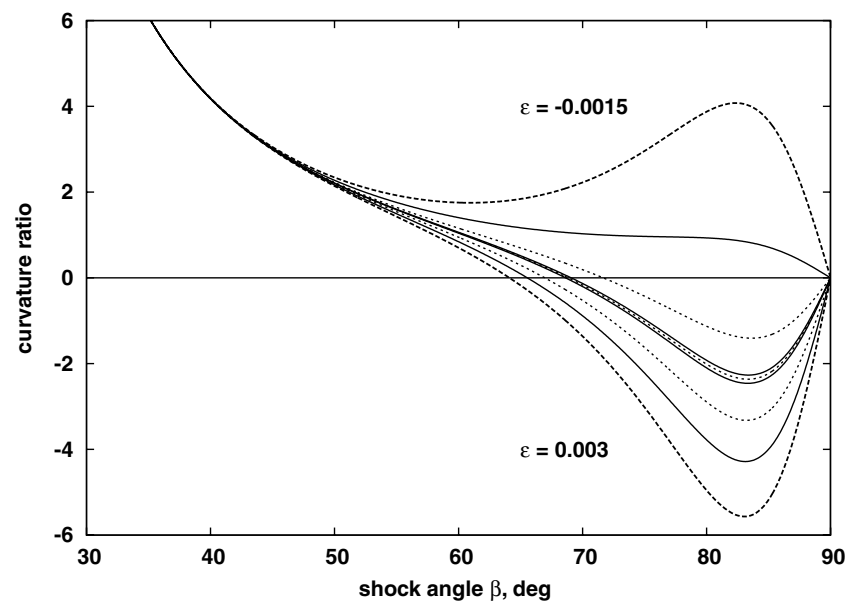

Fig. 9 Ratio of streamline curvature at the shock-to-shock curvature in plane flow. The values of $\varepsilon$ have been chosen differently for this figure: $\varepsilon=0.003,0.005,0.01,0.1,1000,-0.1,-0.01,-0.003$, and -0.0015 , are shown in heavy-broken, full, broken, light-full, light-broken, light-full, broken, full, and heavy-broken lines, respectively. The lowest heavybroken curve corresponds to a high dissociation rate, the light-broken curve corresponds to frozen flow $(r=0)$, and the curves above the lightbroken curve correspond to exothermic reaction $(r<0)$. Hyperbolic shock, $M=6, \gamma=1.4, \theta=0.95$.

exothermic reaction $(\varepsilon<0)$. Qualitatively, the pressure field looks very similar to the density field.

\section{Streamline Curvature}

By differentiating the deflection angle

$$
\delta=\beta-\arctan (v / u)
$$

with respect to $x$ and with respect to $y$ and combining these derivatives to form the derivative of $\delta$ with respect to distance along the streamline, one may obtain the streamline curvature at the shock. Plots of the ratio of streamline curvature to shock curvature are shown in Fig. 9 as functions of $\beta$ for different values of the reaction rate, and for a hyperbolic shock with $M=6, \gamma=1.4$, and $\theta=0.95$.

Observe first the light broken curve for perfect gas flow. At the normal-shock point $\beta=90 \mathrm{deg}$ the streamline curvature is zero. As the shock angle is decreased, the curvature ratio decreases to a minimum and increases to cross to positive values at around $\beta=68 \mathrm{deg}$. This point is called the Crocco point, at which the streamline curvature is zero even with nonzero shock curvature. At smaller

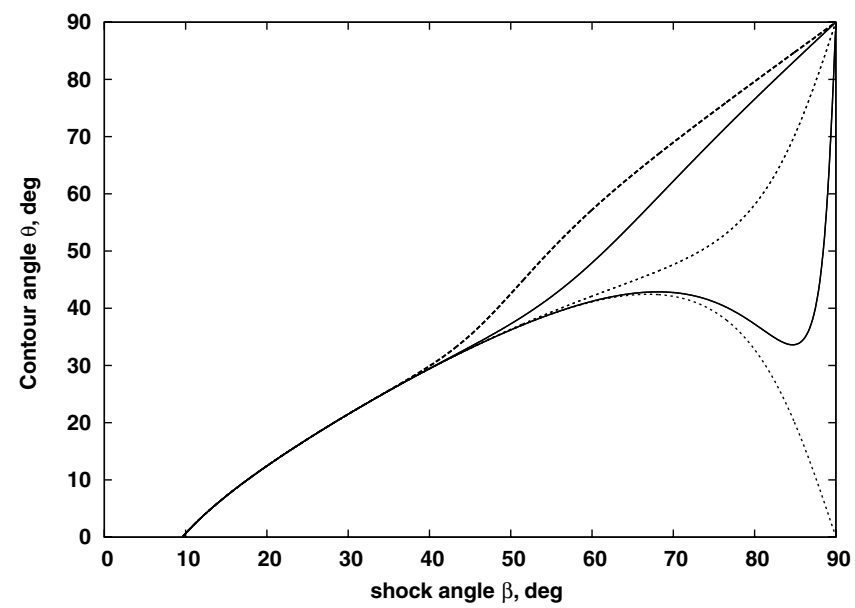

shock angles the ratio remains positive and goes to infinity at the Mach angle. The latter occurs because the shock curvature approaches zero more rapidly than the streamline curvature as $\beta \rightarrow \mu$.

Now consider the case of endothermic reaction $(\varepsilon>0)$. As the reaction rate increases, the minimum value of the ratio decreases, and the Crocco point moves to smaller shock angles. The case of exothermic reaction is more interesting. Here the Crocco point moves to higher shock angles as the reaction rate becomes more negative until, for $\varepsilon$ somewhere between -0.01 and -0.003 (between the upper broken and upper full curves), it disappears entirely, and the ratio is positive for all shock angles. This has interesting implications for the instability of detonation waves that are discussed in Hornung [12]. In that paper the results for streamline curvature are also used to draw conclusions about shock curvatures at a triple shock point. These conclusion need to be reconsidered in light of some new results on viscous effects near triple shock points published by Bondar et al. [21].

\section{Entropy Field}

In the case of the entropy contour it is interesting to plot the angle $\alpha$ of the entropy contour relative to the streamline direction, see the right part of Fig. 10. This is because the entropy is constant along a streamline in frozen flow $(r=0)$. Thus, the light broken curve in the right side of Fig. 10 lies along the line $\alpha=0$. With $r \neq 0$ the entropy increases in flow direction along a streamline independently of whether $r$ is positive or negative. Also, all curves for finite reaction rate merge into the point $(90,90 \mathrm{deg})$, because the contours must be normal to the stagnation streamline for finite $r$. This is also the case in the plot on the left of Fig. 10 showing the angle $\theta$ between the freestream direction and the entropy contour at the shock. In this case the light broken curve for frozen flow is the familiar $\delta(\beta)$ curve for $M=6, \gamma=1.4$. The directions of the entropy contours in plane and axisymmetric flow are the same.

From a rudimentary knowledge of the behavior of streamlines behind a curved shock (or by applying the information contained in Fig. 9) it is now possible to use this information about the angle of the entropy contour to construct a sketch of the entropy field. Such a sketch showing streamlines and entropy contours downstream of a hyperbolic shock in reacting flow is presented in Fig. 11 (left).

Think of the sketch in Fig. 11 (left) to be for hypersonic dissociating flow with a sufficiently fast reaction rate that the flow reaches equilibrium in a distance that is small relative to the shock radius. As may be seen from Fig. 10 the direction of the entropy contour is the same as that of the streamline for small shock angles. This is because, as the shock angle decreases, the dissociation rate becomes very small, and the entropy contour follows the streamline

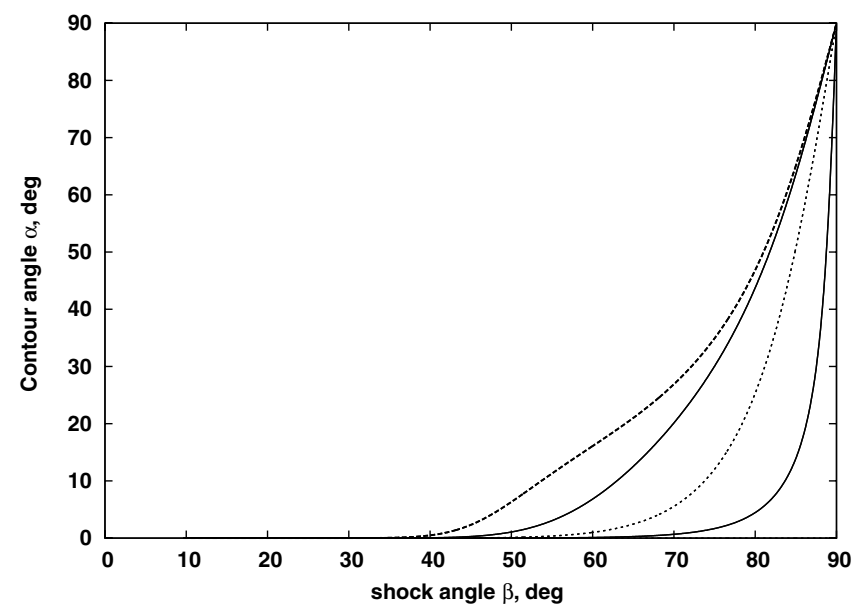

Fig. 10 Entropy contours in plane flow. The left shows the angle between the freestream direction and entropy contour at the shock. The right shows the angle between the entropy contour and streamline direction at the shock. Color key: $\varepsilon=10^{-4}, 10^{-3}, 10^{-2}, 10^{-1}, 10,-10^{-1},-10^{-2},-10^{-3}$, and $-10^{-4}$, in heavy-broken, full, broken, light-full, light-broken, light-full, broken, full, and heavy-broken lines, respectively. Note, however, that only the magnitude and not the sign of $\varepsilon$ affects the entropy contour, as the entropy is increased by finite reaction rate, independently of the sign of $\varepsilon$. Hyperbolic shock, $M=6$, $\gamma=1.4, \theta=0.95$. 


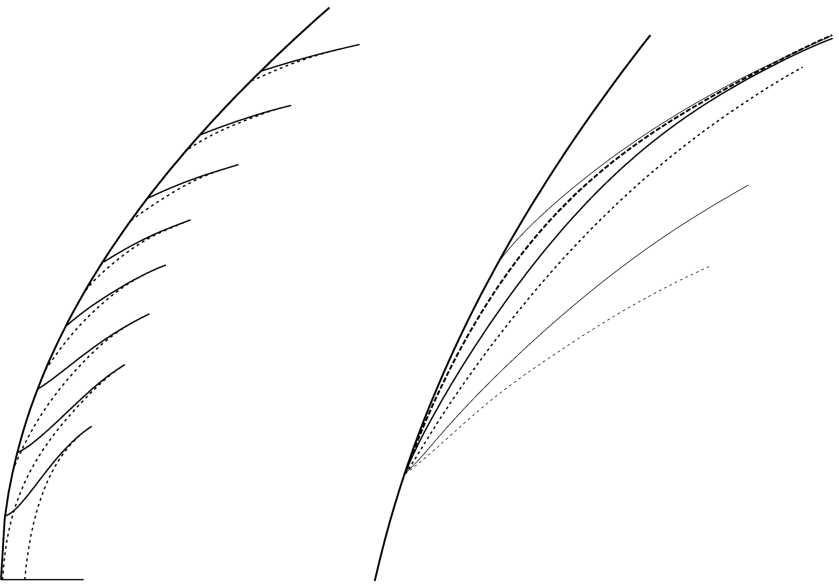

Fig. 11 The left shows equilibrium streamlines (full) and entropy contours (broken) for flow with finite reaction rate downstream of a hyperbolic shock. The streamlines may also be thought of as entropy contours for equilibrium flow. The right shows contours of entropy for a particular value of the entropy, but for different values of $r$, with same line-style code as in Fig. 10. The equilibrium streamline is shown as a full line. Sketches only, $M=\overline{6}$.

as sketched for the uppermost streamline of Fig. 11 (left). Near the stagnation point, the entropy contour is normal to the streamline near the shock, but every entropy contour must asymptotically approach the equilibrium streamline as the flow reaches equilibrium. These conditions, together with a knowledge of the angle of the entropy contour at the shock, give all that is required to sketch the entropy field as shown in Fig. 11 (left).

One can also pick out a particular level of the entropy and sketch the lines along which the entropy has this value for different values of $\varepsilon$. For example, imagine a vertical line in Fig. 10 (left) at $\beta=75 \mathrm{deg}$. This line intersects the lines for different values of $\varepsilon$ at different values of $\theta$. This is shown in Fig. 11 (right). The entropy contours leave the shock from the same point but in different directions. For $r=0$ or $\varepsilon=\infty$, the entropy is constant along the frozen streamline shown as the lower light broken line. As $r$ is increased, the angle increases until the curve for the fast reaction rate $(\varepsilon=0.0001)$ shown as a heavy broken line is almost parallel to the shock. The jump from a fast reaction rate to infinite reaction rate (i.e., to equilibrium flow) manifests itself by the curve for this chosen value of the entropy leaving the shock from a point with smaller shock angle. This is because, at a given shock angle, the entropy jump at the shock for equilibrium flow is larger than for frozen flow. Because the entropy is constant along streamlines in equilibrium flow, the curve for infinite reaction rate (shown in as a full line) is the equilibrium streamline.

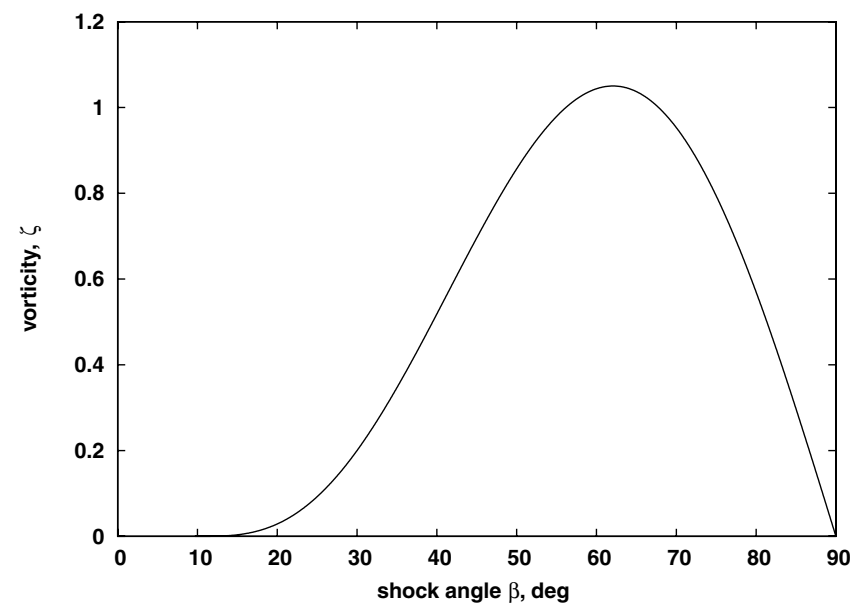

Fig. 12 Vorticity distribution along the shock. Hyperbolic shock, $M=6, \gamma=1.4$.

\section{Vorticity Field}

Define the dimensionless $z$ component of vorticity as $\zeta=$ $\zeta^{\prime} /\left(k_{0}^{\prime} V_{\infty}^{\prime}\right)$ where $\zeta^{\prime}$ is the dimensional $z$ vorticity. The value of $\zeta$ at the shock is then given by

$$
\zeta=k \rho \cos \beta(1-1 / \rho)^{2}
$$

Figure 12 shows the distribution of vorticity over shock angle for a hyperbolic shock and $M=6, \gamma=1.4$. The feature that the vorticity at the shock has a maximum at a shock angle around $60 \mathrm{deg}$ and goes to zero at the stagnation point and at the Mach angle, is typical. However, as may be seen from the dependence of $\zeta$ on $\rho$, one may expect the vorticity to be increased significantly as $\rho$ increases, as is the case for smaller values of $\gamma$ or in endothermic reacting flow.

The equations of Sec. II allow us to determine the direction of the vorticity contour at the shock as a function of $\beta$ and $\varepsilon$. This is shown in Fig. 13 for plane and axisymmetric flow. Consider first the left part of the figure, which is for plane flow. This shows curves only for positive values of $\varepsilon$ in order to keep the graphs from being too confusing. The case of frozen flow (light broken curve) shows that the direction of the vorticity contour is normal to the shock at the stagnation point and, as $\beta$ decreases, $\phi$ increases to a maximum and then decreases to zero at approximately $\beta=62.1 \mathrm{deg}$, where it jumps to $180 \mathrm{deg}$. This, together with the information contained in Fig. 12, allows us to sketch the vorticity contours in the region downstream of a hyperbolic shock as shown in Fig. 14 left.

To check this out, a numerical computation was made using the same scheme as that for Fig. $\underline{4}$, but this time using the fringes to show lines of constant vorticity. This is shown in Fig. 14 (middle).
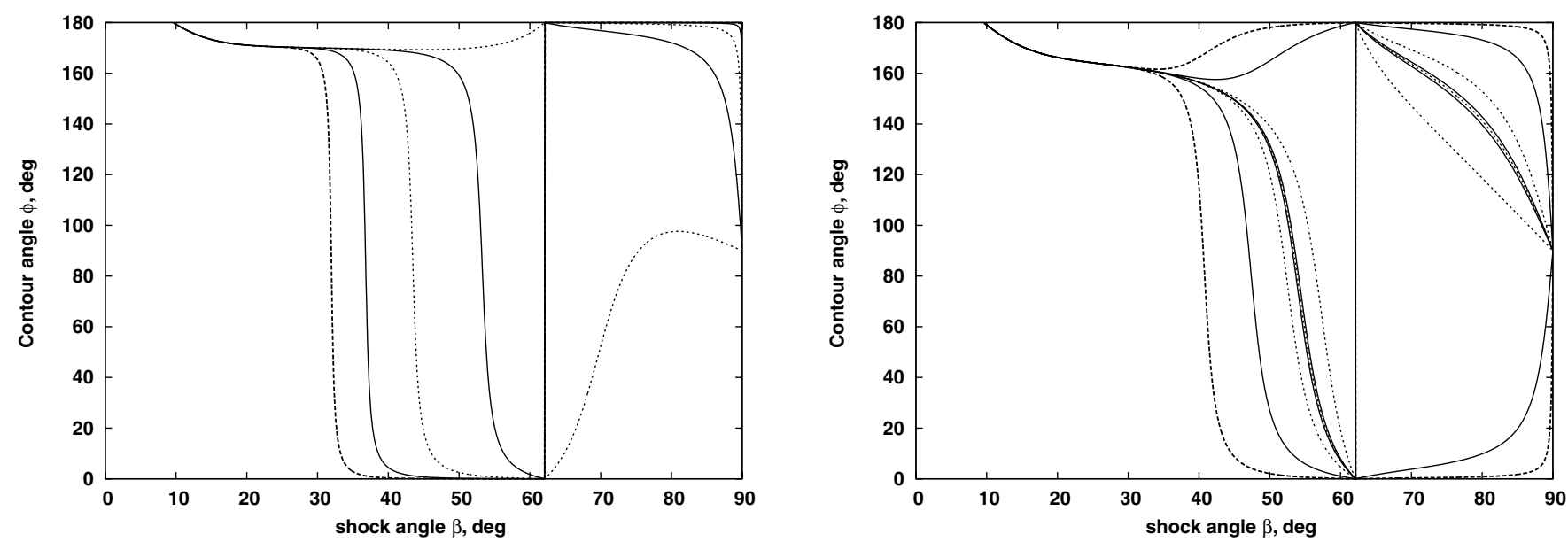

Fig. 13 Angle between vorticity contour and shock. The left shows the plane flow, endothermic reaction only. The right shows the axisymmetric flow with both endothermic and exothermic reacting flow. Hyperbolic shock, $M=6, \gamma=1.4, \theta=0.95$. 

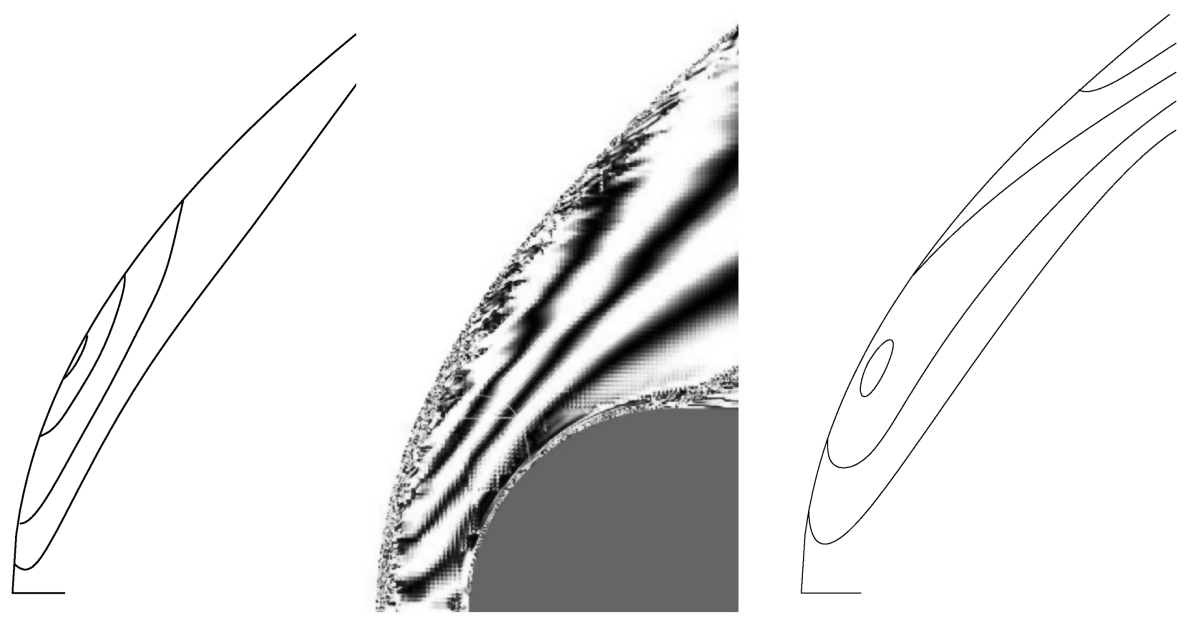

Fig. 14 Vorticity contours in plane flow. The left shows a sketch derived from the distribution of vorticity along the shock (see Fig. 12) and the angle of the frozen-flow vorticity contour at the shock (see light broken curve in Fig. 13, left). The middle shows numerically computed vorticity contours for frozen flow over a circular cylinder. Because the vorticity is obtained by differentiation of discrete information, this plot, in which the fringes are lines of constant vorticity, shows considerable noise. The right shows a sketch of vorticity contours in flow with endothermic reaction derived from Fig. 12 and, say, the full curve in Fig. 13, left. Hyperbolic shock, $M=6, \gamma=1.4$.

Although the differentiation of discrete data necessary to determine the vorticity causes this image to be very noisy, the qualitative agreement between the sketch and the computed flow is evident.

A similar sketch can be made for flow with finite reaction by following, say, the full curve of Fig. 13 (left), for plane flow with endothermic reaction. This is shown in Fig. 14 (right). The vorticity maximum is moved away from the shock and is increased in magnitude, as is consistent with the increase of vorticity expected to accompany the increase in density brought about by the endothermic reaction. This feature is the topic of Hornung and Lemieux [22] who studied the instability of the strong shear layer that is produced by the curved shock when the density ratio rises to values around 14 and above. Their computed vorticity field for dissociating flow is qualitatively very similar to Fig. 14 (right).

It is interesting to observe that the direction of the vorticity contour in axisymmetric flow exhibits a vorticity maximum away from the shock also, even if the flow is frozen, and is qualitatively similar to the endothermic case in plane flow [compare the full curve in Fig. 13 (left) and the light broken curve in Fig. 13 (right)]. The reason for this is that the vortex stretching that occurs in axisymmetric flow acts to increase the vorticity where the streamlines point away from the axis of symmetry. Near the body vortex stretching brings about other interesting features of the vorticity field in axisymmetric flow, see Hayes and Probstein [1].

\section{Conclusions}

A derivation of the shock jump relations for gradients of flow variables is presented. Attention is focused on the special case of a uniform steady freestream with a curved shock that may trigger endothermic or exothermic chemical reaction. The rich information that can be obtained from these is used in order to derive field information for flow variables. The density, pressure, entropy, and vorticity fields, as well as details about the sonic line are discussed. The presentation collects previously published work together with a common notation, and extends it to fields of other variables. Comparisons with experiments and with numerical computations are given in some cases. The gradient jump relations are potentially useful for higherorder numerical schemes that use shock fitting.

\section{Acknowledgments}

This work has benefitted greatly from discussions with my colleague Joseph E. Shepherd and with graduate students to whom I have taught some of the material over many years and on three continents.

\section{References}

[1] Hayes, W. D., and Probstein, R. F., Hypersonic Flow Theory, Academic Press, New York, 1959.

[2] Oswatitsch, K., Gasdynamik, Springer-Verlag, Berlin, 1952.

[3] Lighthill, M. J., "The Flow Behind a Stationary Shock," Philosophical Magazine, Vol. 40, No. 301, 1949, pp. 214-220.

[4] Munk, M. M., and Prim, R. C., "Surface-Pressure Gradient and ShockFront Curvature at the Edge of a Plane Ogive with Attached Shock Front," Journal of the Aeronautical Sciences, Vol. 15, No. 11, 1948, pp. 691-695.

[5] Gerber, N., and Bartos, J. M., "Calculation of Flow-Variable Gradients Behind Curved Shock Waves," Journal of the Aerospace Sciences, Vol. 27, No. 12, 1960, pp. 958-959.

[6] Chen, P. J., and Gurtin, M. J., "Growth and Decay of One-Dimensional Shock Waves in Fluids with Internal State Variables," Physics of Fluids, Vol. 14, No. 6, 1971, pp. 1091-1094. doi:10.1063/1.1693568

[7] Gilinskii, S. M., and Chernyi, G. G., "Supersonic Flow of Combustible Gas Mixture Past Sphere with Account for Ignition Delay Time," Fluid Dynamics, Vol. 3, No. 1, 1968, pp. 12-19. doi:10.1007/BF01016228

[8] Clarke, J. F., Non-Equilibrium Flows Part II, Gasdynamics, edited by P. P. Wegener, Marcel Dekker, New York, 1969.

[9] Mölder, S., "Reflection of Curved Shock Waves in Steady Supersonic Flow," CASI Transactions, Vol. 4, No. 2, 1971, pp. 73-80.

[10] Mölder, S., "Polar Streamline Directions at the Triple Point of Mach Interaction of Shock Waves," CASI Transactions, Vol. 5, No. 2, 1972, pp. 88-89.

[11] Hornung, H. G., "Non-Equilibrium Ideal-Gas Dissociation After a Curved Shock Wave," Journal of Fluid Mechanics, Vol. 74, 1976, pp. 143-159. doi:10.1017/S0022112076001730

[12] Hornung, H. G., "Gradients at a Curved Shock in Reacting Flow," Shock Waves, Vol. 8, 1998, pp. 11-21. doi: $10.1007 / \mathrm{s} 001930050094$

[13] Kaneshige, M. J., and Hornung, H. G., "Erratum: Gradients at a Curved Shock in Reacting Flow," Shock Waves, Vol. 9, 1999, pp. 219-220. doi:10.1007/s001930050158

[14] Hornung, H. G., "Features of the Density Field of Dissociating Hypersonic Flows," Zeitschrift für Angewandte Mathematik und Mechanik, Vol. 80, 2000, pp. 755-763.

doi:10.1002/1521-4001(200011)80:11/12\&lt;755::AID-ZAMM755 $\& \mathrm{gt} ; 3.0 . \mathrm{CO} ; 2-5$

[15] Quirk, J. J., "A Computational Facility (for CFD modelling)," Lecture series (Von Karman Institute for Fluid Dynamics), Vol. 3, 1998, pp. D1-D72.

[16] Billig, F. S., "Shock Wave Shapes Around Spherical—and Cylindrical -Nosed Bodies," Journal of Spacecraft and Rockets, Vol. 4, 1967, pp. 822-823. doi: $10.2514 / 3.28969$

[17] Candler, G. V., "On the Computation of Shock Shapes in Nonequilibrium Hypersonic Flows," 27th Aerospace Sciences Meeting, 
AIAA Paper 89-0312, 1989.

[18] Klomfass, A., Hyperschallströmungen im thermodynamischen Nichtgleichgewicht, Diplom-Ingenieur Thesis, Technical University of Aachen, Aachen, Germany, 1995.

[19] Hornung, H. G., and Smith, G. H., "The Influence of Relaxation on Shock Detachment," Journal of Fluid Mechanics, Vol. 93, 1979, pp. 225-239. doi:10.1017/S0022112079001865

[20] Hornung, H. G., and Leyva, I. A., "Sonic Line and Shock Detachment in Hypervelocity Cone Flow," Transsonicum IV, edited by H. Sobieczky, Kluwer Academic, Norwell, MA, 2003, pp. 363-368.
[21] Bondar, Y. A., Khotyanovsky, D. V., Kudryavtsev, A. N., Shoev, G. V., and Ivanov, M. S., "Viscosity Effects on Mach Reflection in Steady Flows," AIAA Paper 2008-1187, Jan. 2008.

[22] Hornung, H. G., and Lemieux, P., "Shock Layer Instability Near the Newtonian Limit of Hypersonic Flows," Physics of Fluids, Vol. 13, No. 8, 2001, pp. 2394-2402.

doi: $\underline{10.1063 / 1.1383591}$
A. Tumin Associate Editor 